\title{
Depresión, desregulación emocional y estrategias de afrontamiento en adolescentes con conductas de autolesión
}

\author{
Gildardo Bautista Hernández; José Ángel Vera Noriega; \\ Francisco Antonio Machado Moreno; Claudia Karina Rodríguez Carvajal
}

\begin{abstract}
Cómo citar este artículo:
Bautista, G., Vera, J. A., Machado, F. A., \& Rodríguez, C. K. (2022). Depresión, desregulación emocional y estrategias de afrontamiento en adolescentes con conductas de autolesión. Acta Colombiana de Psicología, 25(1), 137-150. https:// www.doi.org/10.14718/ACP.2022.25.1.10
\end{abstract}

Recibido, enero 20/2020; Concepto de evaluación, junio 6/2021; Aceptado, septiembre 14/2021

Gildardo Bautista Hernández

ORCID: https://orcid.org/0000-0001-7670-7705

Universidad Intercultural del Estado de Puebla, Huehuetla, Puebla, México.

José Ángel Vera Noriega ${ }^{1}$

ORCID: https://orcid.org/0000-0003-2764-4431

Centro de Investigación en Alimentación y Desarrollo, A. C., Hermosillo, Sonora, México.

Francisco Antonio Machado Moreno

ORCID: https://orcid.org/0000-0002-5061-2107

Universidad de Sonora, Hermosillo, Sonora, México.

Claudia Karina Rodríguez Carvajal

ORCID: http://orcid.org/0000-0002-4405-6482

TECNOESTATA S. C., Hermosillo, Sonora, México.

Resumen

\begin{abstract}
En México, el estudio de la autolesión cuenta con pocas investigaciones, y menos si se trata de estudios que conformen posibles modelos explicativos. Teniendo esto en cuenta, en el presente trabajo se planteó como objetivo conocer la relación de la autolesión con la depresión, la desregulación emocional y los estilos de afrontamiento, con el fin de generar un modelo explicativo de la problemática. El estudio se realizó a partir de un diseño cuantitativo, transversal y con alcance explicativo, y se contó con una muestra de 5835 adolescentes $\left(M_{\text {edad }}=11-15\right.$ años $)$ inscritos en 62 secundarias públicas del estado de Sonora. Se realizaron regresiones logísticas para el análisis de los datos, y como resultado se encontró que, para los estudiantes con autolesión severa $(3.54 \%)$, el modelo explica el $61 \%$ de la varianza en los datos $\left(R^{2}\right.$ de Nagelkerke $\left.=.61\right)$ y clasifica correctamente al $84.1 \%$ de los participantes; mientras que, en la autolesión leve (5.29\%), el modelo explica el $72 \%$ de la varianza en los datos $\left(R^{2}\right.$ de Nagelkerke $\left.=.72\right)$ y clasifica correctamente al $88.2 \%$ de los estudiantes. Asimismo, las dimensiones de depresión mostraron el mayor nivel explicativo (cognitiva-afectiva, somática) y correlaciones positivas con la desregulación emocional y el estilo de afrontamiento de evitación, así como correlaciones negativas con el estilo de afrontamiento de aproximación. Al final se discute respecto a la importancia de las variables independientes para futuras investigaciones, y sobre los resultados anómalos y otros datos de interés.

Palabras clave: autolesión, depresión, adolescentes, afrontamiento, trastorno emocional.
\end{abstract}

1 Carretera a la Victoria Km 0.6. C. P.: 83304, Hermosillo, Sonora, México. Tel.: +52 (662) 280 0485, ext.: 317. avera@ciad.mx Este estudio fue financiado en parte por la Unidad de Igualdad de Género de la Secretaría de Educación y Cultura del Estado de Sonora, a través de un contrato titulado "Identificación de los factores asociados a la autolesión en jóvenes preadolescentes en escuelas secundarias públicas del Estado de Sonora", con número de registro SEC-CPSP-04-18.

Los autores declaran no tener conflicto de intereses alguno. 


\title{
Depression, emotional dysregulation and coping strategies in adolescents with self-injurious behaviors
}

\begin{abstract}
In Mexico, there is little research on the study of self-injury and even less on possible explanatory models. With this in mind, this study aimed to determine the relationship between self-injury and depression, emotional dysregulation, and coping styles, to generate an explanatory model of the problem. The study was carried out using a quantitative, cross-sectional, explanatory scope, design and a sample of 5835 adolescents (Mage $=11-15$ years) enrolled in 62 public high schools in the state of Sonora. Logistic regressions were performed for data analysis, and as a result it was found that, for students with severe self-injury (3.54\%), the model explains $61 \%$ of the variance in the data (Nagelkerke's R2 $=.61$ ) and correctly classifies $84.1 \%$ of the participants; whereas, in mild self-injury (5.29\%), the model explains $72 \%$ of the variance in the data (Nagelkerke's R2 $=.72$ ) and correctly classifies $88.2 \%$ of the students. Likewise, the depression dimensions showed the highest explanatory level (cognitive-affective, somatic) and positive correlations with emotional dysregulation and avoidance coping style, as well as negative correlations with approach coping style. At the end, the importance of the independent variables for future research is discussed, as well as anomalous results and other data of interest.

Keywords: self-injury, depression, adolescents, coping, emotional disturbance.
\end{abstract}

\section{Introducción}

La autolesión es intencional, autoefectuada y de baja letalidad corporal, tiene daños socialmente inaceptables, y por lo general es realizada por el adolescente para reducir la angustia psicológica que interfiere con su bienestar emocional (Walsh, 2006). Anteriormente, se consideraba que las personas que se cortaban solían tener problemas psiquiátricos como el trastorno límite de la personalidad (Kang et al., 2018), o que provenían de familias con serios problemas disfuncionales o con historial de abuso físico o sexual; no obstante, aunque se ha encontrado que la conducta es más frecuente en personas con este tipo de padecimientos (Vallejo \& Granados, 2017), cada vez es más aceptado que esta conducta se presente también en la población general (Lewis \& Arbuthnott, 2012), especialmente en adolescentes de escuelas secundarias y preparatorias, jóvenes universitarios, y adultos (Albores et al., 2014; Frías et al., 2012; Liu, 2017).

Respecto a la literatura sobre el tema, en un estudio de Whitlock et al. (2006) con una muestra de universitarios que se autolesionaban, el $5 \%$ señaló que inició con esta conducta antes de cumplir los 10 años. De hecho, la edad de inicio encontrada en múltiples estudios ronda entre los 12 y los 16 años (Muehlenkamp \& Gutiérrez, 2004; Whitlock et al., 2006).

En Estados Unidos y Canadá se ha encontrado que la prevalencia de estas conductas en adolescentes se encuentra entre el 13 y el 16\% (Brausch \& Gutiérrez, 2010; Muehlenkamp \& Gutiérrez, 2004; Ross \& Heath, 2002). En los países hispanohablantes, las formas más comunes de autolesión son cortarse $(85 \%)$, pegarse $(32 \%)$ y quemarse (30\%) (Santos, 2006, como se cita en Santos, 2012). En la Ciudad de México, se encontró que el $10 \%$ de los que se autolesionaban lo hacía de manera repetitiva durante al menos un año, y que las personas que lo hacían podían pertenecer a cualquier nivel socioeconómico (Santos, 2008).

En un estudio realizado en Sonora, México, con una muestra de 5835 alumnos de secundarias públicas, la prevalencia fue de $14 \%$ (Vera et al., 2018), y, si se desglosan los datos según el sexo de los estudiantes, esta conducta resultó ser más alta en mujeres (15.6\%) que en hombres (9.2\%); resultados similares a los encontrados por De la Sancha et al. (2020) con estudiantes de secundarias del estado de Guanajuato.

En los últimos 10 años, la problemática ha recibido atención significativa por parte de los investigadores, y solo fue hasta el año 2014 que la autolesión no suicida se 
incluyó dentro del DSM-5 como una problemática que requiere mayor estudio para presentar un modelo explicativo con mayor precisión (Klonsky et al., 2011). De hecho, en una revisión sistemática realizada por Duarte et al. (2021) sobre la autolesión se concluyó que son pocos los estudios existentes sobre modelos que expliquen las causas de dicha problemática.

Ahora bien, para Gratz et al. (2018), la persona que se autolesiona presenta características como la tendencia a la evitación — relacionada con baja tolerancia al estrés-, respuestas emocionales intensas, habilidades deficientes de regulación emocional, y deficiencias en la implementación de estrategias adecuadas cuando se encuentra emocionalmente alterada. No obstante, según Klonsky (2009), la autolesión ayuda a disminuir la intensidad de emociones como frustración, enojo y ansiedad; y las emociones negativas intensas son las que mayormente se regulan practicando la autolesión, en contraste con emociones negativas de poca agitación, como la tristeza y la soledad. De este modo, se ha dicho que la autolesión ayuda a reemplazar aquellas emociones negativas intensas por emociones positivas de poca intensidad, como la calma y el alivio (Klonsky, 2009), y que es una forma desadaptativa de autorregulación emocional (Frías et al., 2012). En otras palabras, se podría afirmar que la autolesión no suicida es un mecanismo utilizado por los adolescentes frente a la incapacidad para regular las emociones y manejar los síntomas de depresión (Peh et al., 2017; Wu et al., 2021).

Respecto a esto último, la asociación entre la autolesión y la depresión ha sido recientemente subrayada por Plener et al. (2015) en una revisión sobre estudios longitudinales que abordan la problemática, y en ella se afirma que, después de episodios pasados de autolesión, la depresión es el primer factor predictivo. Incluso, diversos estudios han encontrado que las personas que se autolesionan puntúan más alto y de manera significativa en depresión en comparación con las que no se autolastiman (Duarte et al., 2021; Khazaie et al., 2020; Ross \& Heath, 2002; You et al., 2016); y autores como Heath et al. (2016) reportan que mayores niveles de depresión pueden predecir a los miembros del grupo que se autolesionan.

Adicional a esto, en un estudio longitudinal con un año de seguimiento realizado por Duggan et al. (2015) se diferenciaron tres grupos con edades entre los 11 y los 13 años: (a) el grupo de no autolesión, que reportó menores niveles de desregulación emocional y síntomas depresivos; (b) el grupo de autolesión en el pasado, que reportó niveles superiores en estas dos variables; y (c) el grupo que se autolesiona actualmente, que reportó mayores niveles que los anteriores para estas dos variables en el segundo corte. En dicho estudio, la desregulación emocional decrementó con el tiempo sin importar el grupo o el género, pero los síntomas depresivos no mostraron cambio alguno.

Asimismo, Marshall et al. (2013), en un estudio longitudinal de un año con tres seguimientos en niños de 13 años en promedio que se encontraban en séptimo grado al inicio del estudio, encontraron que entre el primer y segundo corte la depresión predecía el incremento en la autolesión, mientras que en el segundo a tercer corte esta relación pasaba a ser de coocurrencia, sin tener diferencias entre hombres y mujeres.

Finalmente, Howe-Martin et al. (2012) encontraron en una muestra con 34 adolescentes que reportaron autolesión que estos se distinguían de sus pares por las características de evitación como estrategia de afrontamiento; característica que fue más acentuada en mujeres. Así también, Kirchner et al. (2011) encontraron que las mujeres que se autolesionan puntúan significativamente más alto en las estrategias de análisis lógico, evitación cognitiva, aceptación-resignación y en el total de respuestas de evitación, y significativamente más bajo en la estrategia de resolución de problemas en comparación con las que no se autolesionan; en dicha investigación, los adolescentes de ambos sexos usaron de forma significativa y con mayor frecuencia la estrategia de descarga emocional, por lo que se afirma que los déficits sociales pueden incrementar la intensidad emocional negativa que desemboca en la autolesión (Klonsky et al., 2011).

A partir de los antecedentes expuestos, en el presente estudio se planteó como objetivo identificar la relación entre la autolesión y la depresión, la desregulación emocional y los estilos de afrontamiento, para generar un modelo explicativo de la problemática. Para ello, se plantearon tres hipótesis iniciales: (a) el modelo tendrá niveles explicativos mayores al $25 \%$, debido al consenso académico al respecto; (b) la desregulación emocional será la principal variable explicativa; y (c) los estilos de afrontamiento de aproximación tendrán una relación negativa con la autolesión. 


\section{Método}

Diseño

Se llevó a cabo un estudio de enfoque cuantitativo, no experimental, transversal, y con alcance correlacional-causal, ya que los datos se recabaron en una sola ocasión, no se manipuló deliberadamente las variables independientes, $\mathrm{y}$ se realizaron análisis que buscaron explicar las razones por las cuales los estudiantes se autolesionaban (HernándezSampieri \& Mendoza, 2018).

\section{Participantes}

Se contó con un total de 5835 adolescentes inscritos durante el ciclo escolar 2017-2018 en 62 escuelas secundarias públicas de Sonora, México, ubicadas dentro o en la periferia de colonias que durante el 2016 ocuparon los primeros 10 lugares en reportes de violencia intrafamiliar. El tamaño de muestra representa un número ligeramente mayor al solicitado al calcular la muestra utilizando el $99 \%$ de confianza y $2 \%$ de error de un total de 6473608 estudiantes inscritos en el año 2017 en el sistema educativo público a nivel de secundaria (Dirección General de Planeación, Programación y Estadística Educativa, 2019). En particular, se utilizó un muestreo no probabilístico por cuotas, equilibrando las variables de sexo, grado y turno, y se excluyeron estudiantes de escuelas públicas ubicadas fuera de estas colonias, escuelas privadas, estudiantes que no asistieron a la escuela el día u hora de aplicación, y estudiantes que, si bien aceptaron participar en el estudio, no contestaron a más del $10 \%$ de los reactivos. De los 5835 adolescentes de la muestra total, la mayoría pertenecía al turno matutino (68.4\%), el $50.1 \%$ eran mujeres, y sus edades se ubicaron en un rango de 11 a 15 años, el cual corresponde al rango de edad de inicio de estas conductas.

\section{Instrumentos}

\section{Cédula de datos demográficos}

Se utilizó un cuestionario para recabar información sociodemográfica — edad, sexo, turno, escuela y ciudadque permitió contextualizar la situación de los estudiantes.

\section{Cédula de autolesión (CAL)}

La Cédula de Autolesión (CAL) es un cuestionario desarrollado por Marín (2013) a partir de una revisión exhaustiva de la literatura sobre el tema, para adolescentes en secundarias de México. Se compone de 11 reactivos diseñados para la autoevaluación temporal del último año en que realizaron conductas de autolesión no suicida. Cinco reactivos se consideran para el diagnóstico de autolesión severa y seis de ellos se consideraron como autolesiones leves, por lo que se requiere de información adicional para confirmar un diagnóstico positivo. Los reactivos presentan ejemplos de autolesiones específicas y se contestan con una escala Likert de frecuencia con cinco opciones de respuesta: 1 = " 0 veces", 2 = "1 vez", 3 = "2 a 4 veces", 4 = "5 a 9 veces" y $5=$ "10 o más veces" durante el último año. El punto de corte establecido por el autor es de tres para identificar la autolesión leve, y de cuatro para autolesión severa. Esta cédula es unidimensional, según los resultados del análisis factorial exploratorio para esta muestra, donde se encuentra que el modelo explica el $48.37 \%$ de la varianza. Su fiabilidad, obtenida a través del valor alfa de Cronbach, fue de .89 .

\section{Dificultades en la regulación emocional (DERS-E)}

Se utilizó la adaptación para adolescentes mexicanos desarrollada por Marín et al. (2012) del instrumento Dificultades en la Regulación Emocional (DERS-E) (Gratz $\&$ Roemer, 2004). Este cuestionario contiene 24 reactivos que miden dificultades en la regulación de las emociones, $y$ la versión mexicana está compuesta por cuatro factores: (a) no aceptación de respuestas emocionales, (b) dificultades para implementar conductas dirigidas a metas, (c) falta de consciencia emocional, y (d) falta de claridad emocional. Las opciones de respuestas están formuladas en formato Likert de frecuencia -1 = "casi nunca" a $5=$ "casi siempre"-. Los alfas de Cronbach de las dimensiones oscilan entre .68 y .85 , y en conjunto explicaron el $45.3 \%$ de la varianza. Además, los autores reportaron que el modelo se ajustó aceptablemente a los datos $\left(\chi^{2}=259.72, \mathrm{gl}=230\right.$, $p>.05 ; \mathrm{NFI}=.92 ; \mathrm{RMSEA}=.01<.05)$.

\section{Depresión (BDI-IA)}

Se utilizó el Inventario de Depresión de Beck (BDI) (Beck et al., 1988) adaptado por Beltrán et al. (2012) para población adolescente mexicana (BDI-IA). Este instrumento se compone de 21 reactivos autoaplicables — con cuatro opciones de respuesta - para evaluar la severidad de los síntomas de depresión durante la última semana previa a 
la aplicación. Los autores reportan que el coeficiente de confiabilidad en el BDI-IA con población mexicana muestra oscilaciones entre $\alpha=.68$ y $\alpha=.91$; que la estructura factorial del instrumento se configura en dos dimensiones — dimensión cognitiva-afectiva y dimensión somática-; y que esta estructura obtuvo un ajuste aceptable en el análisis factorial confirmatorio $\left(\chi^{2}=309.59 ; p=.000\right.$; $\mathrm{CMIN}=1.64 ;$ RMSEA $=.042$, IC $90 \%[.03, .05] ; \mathrm{CFI}=.95$; $\mathrm{IFI}=.95 ; \mathrm{GFI}=.92)$.

\section{Afrontamiento a los problemas (CRI-Y Form)}

Se utilizó el instrumento de Respuestas de Afrontamiento para Adolescentes (CRI-Y Form) de Moos (1993), adaptado para población mexicana por Vallejo et al. (2007). Este instrumento consta de 48 ítems que evalúan ocho estrategias de afrontamiento: análisis lógico (AL), revaloración positiva (RP), búsqueda de guía (BG), solución del problema (SP), evitación cognitiva (EVCOV), aceptación/resignación $(\mathrm{A} / \mathrm{R})$, refuerzos alternativos (RA) y descarga emocional (DE).

La aplicación consiste en pedir a los participantes que escriban en una tarjeta un problema que hayan tenido en los últimos doce meses. Posteriormente, se les pide que coloquen sus respuestas en formato Likert pensando en cómo enfrentaron el problema que previamente escribieron —siendo 1 = "No, nunca" y 5 = "Sí, casi siempre"—. Los autores reportaron una fiabilidad de .80 para el instrumento, y que es posible agrupar las ocho dimensiones en dos grandes factores principales: estrategias de acercamiento y estrategias de evitación.

\section{Procedimiento}

Se elaboró un cuadernillo con los instrumentos divididos en dos partes: la primera tenía 11 preguntas relacionadas con los datos contextuales de los estudiantes, y la segunda, cada una de las escalas del estudio. Este cuadernillo se presentó a un grupo de psicólogos para que revisaran las instrucciones y el tiempo que se tomaría para contestar dicho instrumento, para poder solicitar los permisos a las escuelas. La Secretaría de Educación y Cultura envió una carta a los directores de las escuelas seleccionadas para permitir el ingreso a las instituciones, dado que previamente se firmó convenio con esta institución, la cual asumiría la función de informar a la comunidad educativa del estudio, incluyendo a los padres de familia.
Para disminuir los problemas de sesgo, fue necesario estandarizar - a través de reuniones de una hora de duración - a cuatro equipos de aplicadores. La estandarización se enfocó en temas como el conocimiento sobre el proyecto, los instrumentos de medida a aplicar, las instrucciones al momento de la aplicación, el monitoreo durante la aplicación, la revisión de las hojas de respuesta al momento de la entrega, y la organización de las hojas de respuesta.

Por tratarse de adolescentes, se solicitó por escrito el consentimiento informado nacional a los padres o tutores, además del consentimiento firmado por los adolescentes. En el consentimiento se les informaba, a los adolescentes y a sus padres, que no habría beneficios financieros relacionados con su participación, y que los datos recolectados serían resguardados en el anonimato y la secrecía de la información, por lo que al contestar quedaba claro cuáles eran los propósitos del estudio, la seguridad, la confidencialidad de la información y el carácter voluntario de su participación. El proyecto fue revisado y aprobado por la Secretaría de Educación y Cultura del Estado de Sonora a través de la Unidad de Igualdad de Género.

Habiendo obtenido el consentimiento informado, se acudió a los planteles para aplicar los instrumentos, con previa autorización de los directores y profesores de las escuelas. Al confirmar los permisos respectivos, se procedió a realizar la evaluación, y se entregó el instrumento, dando una explicación sobre el objetivo de la investigación y la manera en que se debía contestar. La aplicación del instrumento se realizó de forma grupal y tuvo una duración de entre 40 y 80 minutos. Dicha aplicación se alineó a los estándares éticos vigentes, y contó con el consentimiento informado de directores, docentes, padres y de los mismos estudiantes participantes, acorde con los artículos 118 y 122 del Código ético del psicólogo de la Sociedad Mexicana de Psicología (2007). Por otra parte, la investigación se basó en el artículo 136 de dicho código ético, que hace referencia a la confidencialidad, ya que se informó acerca de la utilización de los datos recopilados, así como el uso anónimo de los mismos.

Cabe señalar que al término del estudio, y con base en los datos obtenidos, los docentes de las escuelas participantes en el estudio recibieron capacitación sobre las recomendaciones y medidas de actuación que tienen como objetivo prevenir que el comportamiento autolesivo progrese e interfiera con 
el desarrollo emocional, personal y académico del alumno. Finalmente, se entregó a la Secretaría de Educación el listado de alumnos con autolesión severa para que los directivos y docentes pudieran, junto a los padres y personal de apoyo, darles oportunidades de solución o canalizarlos a servicios de salud mental para su recuperación.

\section{Aspectos éticos}

El presente artículo se deriva de un proyecto financiado por la Secretaría de Educación y Cultura del Estado de Sonora, a través de la Unidad de Igualdad de Género, titulado "Identificación de los factores asociados a la autolesión en jóvenes preadolescentes en escuelas secundarias públicas del Estado de Sonora", con número de registro SEC-CPSP-04-18, por lo que se tuvieron todos los cuidados éticos posibles. Por tratarse de adolescentes, se solicitó el consentimiento informado por escrito a los padres o tutores, además del consentimiento firmado por los adolescentes. En el consentimiento se les informaba, a los adolescentes y a sus padres, que no habría beneficios financieros relacionados con su participación, y que los datos recolectados serían resguardados en el anonimato y la secrecía de la información, por lo que al contestar quedaba claro cuáles eran los propósitos del estudio, la seguridad, la confidencialidad de la información y el carácter voluntario de su participación.

\section{Análisis estadísticos}

Durante el procedimiento de análisis estadísticos, primero se sustituyó - por la media del grupo- el $2 \%$ de datos perdidos por enmendaduras en las hojas electrónicas o por falla del lector óptico. Posteriormente, se construyeron los grupos con autolesión y sin autolesión a partir de las respuestas que emitieron los estudiantes en la cédula de autolesión. El punto de corte se fijó en 3 para identificar la autolesión leve, y en 4 para autolesión severa (Marín, 2013).

De acuerdo con esto, de los 5835 adolescentes de la muestra total, 207 estudiantes (3.54\%) se clasificaron con conductas de autolesión severa, y 309 (5.29\%) con conductas de autolesión leve. Para cada una de las submuestras, se igualaron con estudiantes que no presentaron este tipo de conductas de autolesión (véase Figura 1). Posteriormente, se realizaron análisis de regresiones logísticas con el método Step Wise para estimar las probabilidades mediante odds ratio (OR) con intervalos de confianza (IC) del $95 \%$, y con un nivel de significancia menor o igual a .05 . Se utilizó el coeficiente $R^{2}$ de Nagelkerke para analizar la varianza explicada del modelo.

Las regresiones logísticas se realizaron primero considerando hombres y mujeres, después solo para la muestra de hombres, $y$, finalmente, solo para la muestra de mujeres. Todo lo anterior se realizó en el Programa Estadístico para las Ciencias Sociales (SPSS), versión 22.

\section{Resultados}

Se realizó un análisis de regresión logística con el método de Step Wise para los adolescentes que fueron clasificados previamente con autolesión leve y sin autolesión leve. La probabilidad de presentar autolesiones leves se asoció positivamente con la depresión cognitivo-afectiva $(\mathrm{OR}=5.41$; IC $95 \%[2.98,9.82] ; p \leq .001)$ y somática $(\mathrm{OR}=2.44$; IC $95 \%$ $[1.45,4.11] ; p=.001)$, con la desregulación emocional —relacionada con la no aceptación- ( OR $=1.91$; IC $95 \%$ [1.31, 2.79]; $p=.001)$, con el afrontamiento centrado en la descarga emocional (OR $=1.50$; IC $95 \%[1.14,1.97]$; $p=.004)$, y con la resignación ( $\mathrm{OR}=1.63$; IC $95 \%$ [1.14, $2.31] ; p=.007) ; \mathrm{y}$ se asoció negativamente con el estilo de afrontamiento centrado en la solución de problemas (OR=-.29; IC $95 \%[.56, .98] ; p=.04)$.

De las variables que se asociaron significativamente, las que tienen mayor probabilidad de explicar la autolesión leve fueron las dos dimensiones de depresión, seguidas de no aceptación y resignación (véase Tabla 1). Este modelo explica el $72 \%$ de la varianza $\left(R^{2}\right.$ de Nagelkerke $\left.=.72\right)$ y clasifica correctamente al $88.2 \%$ de los estudiantes, con mayor porcentaje en adolescentes sin autolesión leve (89.0 \%) que en los de autolesión leve (87.4\%).

Posteriormente, se realizó el análisis de regresión logística separando a hombres y mujeres que fueron clasificados previamente con autolesión leve y sin autolesión leve. En el modelo de hombres, que puede observarse en la Tabla 2, la probabilidad de presentar autolesiones leves se asoció positivamente con la depresión cognitivo-afectiva y somática, con la resignación y con la claridad emocional; mientras que, en el caso de las mujeres, se asoció positivamente con la dimensión cognitivo-afectiva de la depresión, la no aceptación y la descarga emocional, y negativamente con la revaloración positiva - que forma parte de un estilo de afrontamiento de aproximación-. 
Figura 1.

Distribución de las muestras general y especifica para autolesión leve y severa

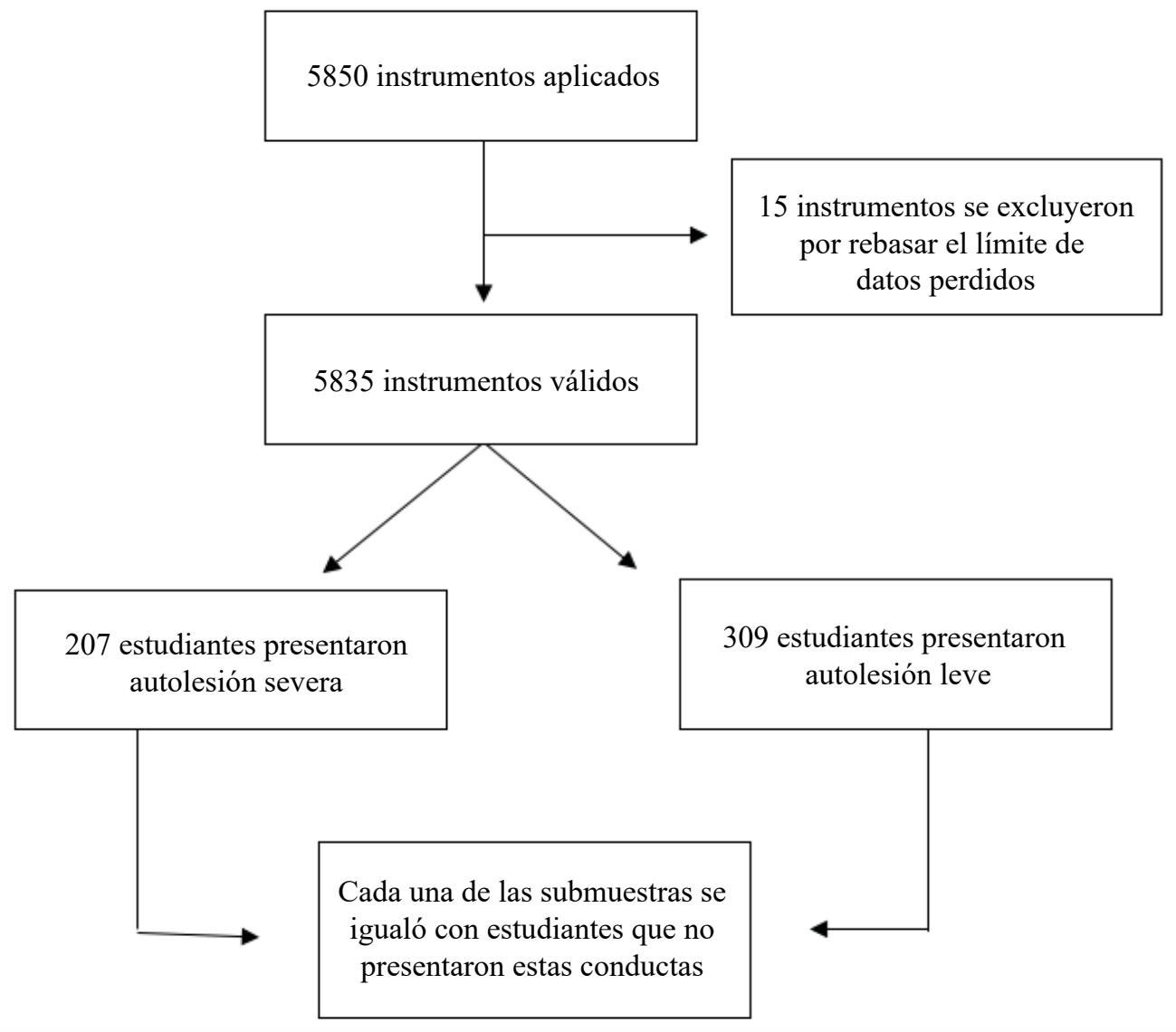

Tabla 1.

Resumen del análisis de regresión logística, prediciendo autolesión leve

\begin{tabular}{lcccccc}
\hline Predictores & B & BE & OR & IC 95 \% & Wald & $p$ \\
\hline Afrontamiento & & & & & & \\
$\quad$ Solución de problemas & -0.29 & 0.14 & 0.75 & {$[0.56,0.98]$} & 4.17 & .04 \\
$\quad$ Descarga emocional & 0.41 & 0.14 & 1.50 & {$[1.14,1.97]$} & 8.37 & .004 \\
$\quad$ Resignación & 0.49 & 0.18 & 1.63 & {$[1.14,2.31]$} & 7.39 & .007 \\
$\quad \begin{array}{l}\text { Desregulación emocional } \\
\quad \text { No aceptación }\end{array}$ & 0.65 & 0.19 & 1.91 & {$[1.31,2.79]$} & 11.30 & .001 \\
$\quad \begin{array}{l}\text { Depresión } \\
\quad \text { Cognitivo-afectiva }\end{array}$ & 1.69 & 0.30 & 5.41 & {$[2.98,9.82]$} & 30.79 & $<.001$ \\
$\quad$ Somática & 0.89 & 0.27 & 2.44 & {$[1.45,4.11]$} & 11.30 & .001 \\
$\quad$ Constante & -7.69 & 0.71 & 0.00 & 0.000 & 116.86 & \\
\hline
\end{tabular}

Nota $. \mathrm{B}=$ beta, $\mathrm{BE}=$ beta estandarizada, $\mathrm{OR}=$ odds ratios, $\mathrm{IC}=$ intervalo de confianza, $p=$ significancia estadística. 
Tabla 2.

Resumen de análisis de regresión logística, prediciendo autolesión leve en hombres y mujeres

\begin{tabular}{|c|c|c|c|c|c|c|c|}
\hline & Predictores & B & $\mathrm{BE}$ & OR & IC $95 \%$ & Wald & $p$ \\
\hline \multirow{5}{*}{ Hombres } & Resignación & 1.06 & 0.28 & 2.88 & {$[1.67,4.98]$} & 14.38 & $<.001$ \\
\hline & Claridad emocional & 0.54 & 0.20 & 1.72 & {$[1.16,2.54]$} & 7.24 & .007 \\
\hline & Cognitivo-afectiva & 1.66 & 0.38 & 5.27 & {$[2.52,11,04]$} & 19.41 & $<.001$ \\
\hline & Somática & 1.17 & 0.36 & 3.24 & {$[1.60,6,53]$} & 10.76 & .001 \\
\hline & Constante & -9.00 & 1.00 & 0.00 & & 81.03 & $<.001$ \\
\hline \multirow{5}{*}{ Mujeres } & Revaloración positiva & -0.51 & 0.200 & 0.59 & {$[0.40,4.98]$} & 6.67 & .01 \\
\hline & Descarga emocional & 0.54 & 0.201 & 1.71 & {$[1.15,2.54]$} & 7.23 & .007 \\
\hline & No aceptación & 0.84 & 0.250 & 2.33 & {$[1.43,11.04]$} & 11.43 & .001 \\
\hline & Cognitivo-afectiva & 2.28 & 0.411 & 9.84 & {$[4.39,6.53]$} & 30.89 & $<.001$ \\
\hline & Constante & -6.19 & 0.923 & 0.002 & & 45.06 & $<.001$ \\
\hline
\end{tabular}

Nota . $\mathrm{B}=$ beta, $\mathrm{BE}=$ beta estandarizada, $\mathrm{OR}=$ odds ratios, $\mathrm{IC}=$ intervalo de confianza, $p=$ significancia estadística.

Específicamente, el modelo de hombres explica el $73 \%$ de la varianza $\left(R^{2}\right.$ de Nagelkerke $\left.=.73\right)$ y clasifica correctamente al $89.9 \%$ de los estudiantes, con mayor porcentaje de aciertos en los adolescentes sin autolesión leve (93.4\%) que en los de autolesión leve (85.0\%); mientras que el modelo de mujeres explica el $71 \%$ de la varianza $\left(R^{2}\right.$ de Nagelkerke $=.71)$ y clasifica correctamente al $85.8 \%$ de las estudiantes, con menor porcentaje de aciertos en las mujeres clasificadas previamente sin autolesión leve (83.8 \%) que en las de autolesión leve (87.3\%).

Por otra parte, se realizó un análisis de regresión logística con el método de Wald para los adolescentes que fueron clasificados previamente con autolesión severa y sin autolesión severa. Tal como se muestra en la Tabla 3, la probabilidad de presentar autolesiones severas se asoció positivamente con la depresión cognitivo-afectiva ( $\mathrm{OR}=2.92$; IC $95 \%[1.72$, $4.96] ; p \leq .001)$ y somática (OR $=2.89 ;$ IC $95 \%[1.72,4.87]$; $p \leq .001)$, con la desregulación emocional — relacionada con la no aceptación- $(\mathrm{OR}=1.51$; IC $95 \%$ [1.02, 2.22]; $p=.03)$ y con la resignación ( $\mathrm{OR}=1.65$; IC $95 \%[1.15$, $2.36] ; p=.008$ ). Este modelo explica el $61 \%$ de la varianza $\left(R^{2}\right.$ de Nagelkerke $\left.=.61\right)$, clasifica correctamente al $84.1 \%$ de los estudiantes, y tuvo mayor probabilidad de acierto en los adolescentes con autolesión severa (84.5\%) que en los adolescentes sin autolesión severa (83.6\%).

Finalmente, se realizó el análisis de regresión logística, separando a hombres y mujeres que fueron clasificados previamente con autolesión severa y sin autolesión severa. En el modelo de hombres, la probabilidad de presentar autolesiones severas se asoció positivamente con la depresión

Tabla 3.

Resumen de análisis de regresión logística, prediciendo autolesión severa

\begin{tabular}{lcccccc}
\hline Predictores & B & BE & OR & IC 95 \% & Wald & $p$ \\
\hline $\begin{array}{l}\text { Afrontamiento } \\
\quad \text { Resignación }\end{array}$ & 0.50 & 0.18 & 1.65 & {$[1.15,2.36]$} & 7.66 & .008 \\
$\quad \begin{array}{l}\text { Desregulación emocional } \\
\quad \text { No aceptación }\end{array}$ & 0.41 & 0.19 & 1.51 & {$[1.02,2.22]$} & 4.411 & .03 \\
$\quad \begin{array}{l}\text { Depresión } \\
\quad \text { Cognitivo-afectiva }\end{array}$ & 1.07 & 0.27 & 2.92 & {$[1.72,4.96]$} & 15.84 & $<.001$ \\
$\quad$ Somática & 1.06 & 0.26 & 2.89 & {$[1.72,4.87]$} & 16.12 & $<.001$ \\
$\quad$ Constante & -6.22 & 0.57 & 0.002 & & 117.92 & \\
\hline
\end{tabular}

Nota $. \mathrm{B}=$ beta, $\mathrm{BE}=$ beta estandarizada, $\mathrm{OR}=$ odds ratios, $\mathrm{IC}=$ intervalo de confianza, $p=$ significancia estadística. 
cognitivo-afectiva y somática, la búsqueda de apoyo, la resignación y las metas, y negativamente con el análisis lógico. En el caso de las mujeres, se asoció con la dimensión cognitivo-afectiva y somática de la depresión, la no aceptación y la resignación (véase Tabla 4).

El modelo de hombres explica el $65 \%$ de la varianza $\left(R^{2}\right.$ de Nagelkerke $=.65)$ y clasifica correctamente al $83.7 \%$ de los estudiantes, con mayor probabilidad de acierto en los adolescentes clasificados sin autolesión severa (84.3\%) que en los de autolesión severa (83.2\%); mientras que el modelo de mujeres explica el $65 \%$ de la varianza $\left(R^{2}\right.$ de Nagelkerke $=.71)$ y clasifica correctamente al $84.9 \%$ de las estudiantes, con mayor probabilidad de acierto en las mujeres clasificadas previamente sin autolesión severa $(85.7 \%)$ que en las de autolesión severa $(84.0 \%)$.

\section{Discusión}

El alto nivel de explicación de varianza $-72 \%$ en autolesión leve y $65 \%$ en autolesión severa - señala la fuerte y profunda relación que la autolesión tiene con las variables estudiadas, razón por la cual se encuentra evidencia empírica de la primera hipótesis planteada. De este modo, se tiene entonces un modelo con buenas implicaciones para ser utilizado en intervenciones psicológicas dentro de las escuelas secundarias participantes, ya que permite apuntar a un alto grado de la población con esta situación.
La segunda hipótesis no fue corroborada, debido a que la desregulación emocional no se encuentra como la principal variable explicadora, lo cual sería un consenso en la literatura (Gratz \& Roemer, 2008; Klonsky, 2009; Robles et al., 2006); y aunque sí resulta positiva la relación con la autolesión en sus diferentes dimensiones, es considerablemente menor a las dimensiones de depresión somática y cognitivo-afectiva, encontradas como los principales indicadores de la conducta en todos los análisis realizados. Recordando los estudios longitudinales de Marshall et al. (2013) y Duggan et al. (2015), la depresión se mantiene a lo largo del tiempo; es interesante observar cómo en este último estudio la desregulación sí disminuye con el tiempo, probablemente por la misma práctica de la autolesión, que ayuda al individuo, precisamente, a regular estos estados emocionales agobiantes, sin que estos, viendo los indicadores de depresión, realmente disminuyan.

Otra explicación respecto a la baja capacidad explicativa de la desregulación emocional podría ser la rumiación cognitiva, pues se ha identificado que la rumiación podría explicar la aparición de los problemas depresivos y las conductas impulsivas; siendo así una manera disfuncional de regular las emociones (Graham et al., 2018; Joormann \& Gotlib, 2010; Liverant et al., 2011).

Por otra parte, las diferencias entre hombres y mujeres en las otras dimensiones significativas son relevantes, dado que por mucho tiempo se ha hablado de la diferencia en prevalencia o en características tales como las motivaciones

Tabla 4.

Resumen de análisis de regresión logística prediciendo autolesión severa en hombres y mujeres

\begin{tabular}{|c|c|c|c|c|c|c|c|}
\hline & Predictores & B & $\mathrm{BE}$ & OR & IC $95 \%$ & Wald & $p$ \\
\hline \multirow{7}{*}{ Hombres } & Búsqueda de apoyo & 0.80 & 0.29 & 2.23 & {$[1.25,3.97]$} & 7.46 & .006 \\
\hline & Análisis lógico & -0.98 & 0.29 & 0.37 & {$[0.21,0.65]$} & 11.55 & .001 \\
\hline & Resignación & 0.67 & 0.26 & 1.95 & {$[1.15,3.30]$} & 6.31 & .01 \\
\hline & Metas & 0.55 & 0.26 & 1.74 & {$[1.03,2.93]$} & 4.34 & .03 \\
\hline & Cognitivo afectivo & 1.14 & 0.40 & 3.13 & {$[1.40,6.96]$} & 7.84 & .005 \\
\hline & Somático & 1.21 & 0.43 & 3.52 & {$[1.60,7.76]$} & 9.81 & .002 \\
\hline & Constante & -6.49 & 1.00 & 0.002 & & 42.26 & $<.001$ \\
\hline \multirow{5}{*}{ Mujeres } & Resignación & 0.62 & 0.27 & 1.873 & {$[0.40,4.98]$} & 5.06 & .024 \\
\hline & No aceptación & 0.55 & 0.26 & 1.733 & {$[1.15,2.54]$} & 4.43 & .035 \\
\hline & Cognitivo afectivo & 1.10 & 0.43 & 3.028 & {$[1.43,11.04]$} & 6.63 & .010 \\
\hline & Somático & 0.93 & 0.39 & 2.552 & {$[4.39,6.53]$} & 5.55 & .018 \\
\hline & Constante & -6.94 & 0.91 & 0.001 & & 57.94 & $<.001$ \\
\hline
\end{tabular}

Nota . $\mathrm{B}=$ beta, $\mathrm{BE}=$ beta estandarizada, $\mathrm{OR}=$ odds ratios, $\mathrm{IC}=$ intervalo de confianza, $p=$ significancia estadística. 
para con la conducta y la duración, el método y el lugar del cuerpo de la autolesión (Laye-Gindhu \& Schonert-Reichl, 2005), pero son algunas excepciones las que estudian las diferencias en las características psicológicas entre los géneros (Klonsky et al., 2003). Futuros estudios serán necesarios, ya que podrán indicar especificidades por sexo para las intervenciones clínicas.

De otro lado, se encontró relación con los estudios de Howe-Martin et al. (2012) y Kirchner et al. (2011), donde dimensiones de afrontamiento evitativo resultaron significativas con los grupos de autolesión, principalmente resignación $(\mathrm{A} / \mathrm{R})$. Cabe mencionar que la particularidad femenina en tendencia evitativa de los estudios citados no se replicó, pues tanto hombres como mujeres mostraron este tipo de afrontamiento.

En el otro extremo, se encontró que las dimensiones revaloración positiva (RP) en mujeres pertenecientes a la autolesión leve, y análisis lógico (AL) en hombres pertenecientes a autolesión severa, cumplen con la tercera hipótesis, es decir, tienen una relación negativa con la autolesión. Existen implicaciones de intervención en estos resultados, ya que señalan que potencialmente el trabajo con estas variables cognitivas - y no conductuales - tendría mayor aprovechamiento en prevenir y ayudar a las personas que se autolesionan.

Como resultado contradictorio, destaca la relación positiva de la dimensión búsqueda de apoyo (BA) en hombres con autolesión severa; siendo una estrategia de afrontamiento de revaloración social, se esperaba que fuera más frecuente en mujeres, porque su desarrollo requiere de comportamiento expresivo social, más probable en mujeres (Ibarra et al., 2002). El adolescente en su mayoría busca apoyo en sus pares, y culturalmente las estrategias consideradas masculinas tienen mayor consideración de evitación, por lo que se teoriza que, al encontrarse con estos patrones de conducta, se tiene un efecto positivo en la agitación emocional y en la autolesión.

Finalmente, como limitantes del estudio es importante mencionar que la obtención de los datos se realizó por medio de autorreportes, lo cual podría elevar la prevalencia en comparación de métodos más exhaustivos como la entrevista a profundidad. También, el hecho de que las escuelas se definieron a partir del alto grado de violencia intrafamiliar que reportaban las colonias circundantes pudo haber tenido un efecto de aumento en las medidas aplicadas.
Por lo anterior, se recomienda seguir investigando sobre este tema con otras metodologías y otras poblaciones, como en escuelas privadas o escuelas que están fuera de los polígonos de violencia.

A partir de nuestros resultados, podemos afirmar que las intervenciones clínicas basadas en las características depresivas son las más viables para tener un buen impacto en la población atendida. Una opción es la Psicoterapia Interpersonal para Depresión en Adolescentes Adaptada para Autolesión (IPTASI), desarrollada por Jacobson y Mufson (2012), que apunta a ayudar a los usuarios a mejorar su relación interpersonal, al incrementar su conciencia y entendimiento emocional, enseñándoles comunicación y habilidades de resolución de problemas mediante técnicas didácticas y de apoyo. Otra opción es la técnica de mindfulness para el tratamiento de padecimientos (Heath et al., 2016), y, finalmente, es de destacar la terapia de perspectiva temporal (Oyanadel et al., 2021), que utilizada en ideación suicida podría reducir los episodios depresivos, de estrés y de ansiedad frecuentes en la autolesión (De Oliveira et al., 2020).

\section{Referencias}

Albores, L., Méndez, J., García, A., Delgadillo, Y., Chávez, C., \& Martínez, O. (2014). Autolesiones sin intención suicida en una muestra de niños y adolescentes de la ciudad de México. Actas Psiquiátricas Españolas 42(4), 159-68. https://www.actaspsiquiatria.es/reposito rio/16/90/ESP/16-90-ESP-159-168-346514.pdf

Beck,A.T., Steer, R.A., \& Garbin, M. G. (1988).Psychometric properties of Beck Depression Inventory: Twenty-five years of evaluation. Clinical Psychology Review, 1(8), 77-100. https://doi.org/10.1016/0272-7358(88)90050-5

Beltrán, M., Freyre, M., \& Hernández, L. (2012). El Inventario de Depresión de Beck: Su validez en población adolescente. Terapia Psicológica, 30(1), 5-13. http://dx.doi.org/10.4067/S0718-48082012000100001

Brausch, A., \& Gutierrez, P. (2010). Differences in NonSuicidal Self-Injury and Suicide Attempts in Adolescents. Journal Youth Adolescence, 39(3), 233-242. https://doi. org/10.1007/s10964-009-9482-0 
De la Sancha, V. E., Meraz, M. E., Cervera, D. C., Martí, R. M., \& Colorado, H. J. L. (2020). Autolesiones en estudiantes de Secundaria: incidencia y factores circundantes. Apuntes de Psicología, 38(1), 23-33. http://apuntesdepsi cologia.es/index.php/revista/article/view/852/551

De Oliveira Pimentel, F., Della Méa, C. P., \& Dapieve Patias, N. (2020). Víctimas de bullying, síntomas depresivos, ansiedad, estrés e ideación suicida en adolescentes. Acta Colombiana de Psicología, 23(2), 205-240. https://doi. org/10.14718/ACP.2020.23.2.9

Dirección General de Planeación, Programación y Estadística Educativa. (2019). Principales Cifras del Sistema Educativo Nacional 2018-2019. SEP.

Duarte Tánori, K. G., Vera Noriega, J. Á., \& Fregoso Borrego, D. (2021). Factores contextuales relacionados a las conductas de autolesión no suicidas: una revisión sistemática. Psicología y Salud, 31(1), 51-59. https://doi. org/10.25009/pys.v31i1.2675

Duggan, J., Heath, N., \& Hu, T. (2015). Non-suicidal self-injury maintenance and cessation among adolescents: a one-year longitudinal investigation of the role of objectified body consciousness, depression and emotion dysregulation. Child and Adolescent Psychiatry and Mental Health, 9(21). https://doi.org/10.1186/ s13034-015-0052-9

Frías, Á., Vázquez, C. M., Del Real, P, Á., Sánchez, C., \& Giné, S. (2012). Conducta autolesiva en adolescentes: prevalencia, factores de riesgo y tratamiento. Cuadernos de Medicina Psicosomática y Psiquiatría de Enlace, 103, 33-48. http://www.editorialmedica.com/download. php?idart $=468$

Graham, B. M., Denson, T. F., Barnett, J., Calderwood, C., \& Grisham, J. R. (2018). Sex hormones are associated with rumination and interact with emotion regulation strategy choice to predict negative affect in women following a sad mood induction. Frontiers in Psychology, 9, 937. https://doi.org/10.3389/fpsyg.2018.00937
Gratz, K., \& Roemer, L. (2004). Multidimensional assessment emotion regulation and dysregulation: development, factor structure and initial validation of the difficulties in emotion regulation scale. Journal of Psychopathology and Behavioral Assessment, 26(1), 41-54. https://doi. org/10.1023/B:JOBA.0000007455.08539.94

Gratz, K., \& Roemer, L. (2008). The relationship between emotion dysregulation and deliberate self-harm among female undergraduate students at an urban commuter university. Cognitive Behaviour Therapy, 37(1), 14-25. https://doi.org/10.1080/16506070701819524

Gratz, K. L., Tull, M. T., Dixon-Gordon, K., Turner, B., \& Chapman, A. (2018). Is the association of deliberate self-harm with emotional relief stable or dependent on emotional context? Journal of Behavior Therapy and Experimental Psychiatry, 60, 61-68. https://doi.or$\mathrm{g} / 10.1016 / \mathrm{j} . j b t e p .2018 .03 .003$

Heath, N., Carsley, D., De Riggi, M. Mills, D., \& Mettler, J. (2016). The relationship between mindfulness, depressive symptoms, and non-suicidal self-injury amongst adolescents. International Academy for Suicide Research, 20(4), 635-649. https://doi.org/10.1080/13811118.2016.1162243

Hernández-Sampieri, R., \& Mendoza, C. (2018). Metodología de la investigación. Las rutas cuantitativa, cualitativa y mixta (1. ${ }^{\mathrm{a}}$ ed.). Editorial Mc Graw Hill Education.

Howe-Martin, L., Murrell, A., \& Guarnaccia, C. (2012). Repetitive nonsuicidal self-injury as experiential avoidance among a community sample of adolescents. Journal of Clinical Psychology, 68(7), 809-829. https:// doi.org/10.1002/jclp.21868

Ibarra, E. P., Laborín, J. F., \& Vera, J. A. (2002) Rasgos de masculinidad-feminidad en la población que habita el desierto del Noroeste de México. Avances en Psicología Clínica Latinoamericana, 20, 45-56. https://www.re searchgate.net/publication/289376719_Rasgos_de_mas culinidad-feminidad_en_la_poblacion_que_habita_el_ desierto_del_Noroeste_de_Mexico 
Jacobson, C., \& Mufson, L. (2012). Interpersonal psychotherapy for depressed adolescents adapted for self-injury (IPT-ASI): rationale, overview, and case summary. American Journal of Psychotherapy, 66(4), 349-74. https://doi.org/10.1176/appi.psychotherapy.2012.66.4.349

Joormann, J., \& Gotlib, I. H. (2010). Emotion regulation in depression: relation to cognitive inhibition. Cognition and Emotion, 24(2), 281-298. https://doi. org/10.1080/02699930903407948

Kang, N., Jiang, Y., Ren, Y., Gong, T., Liu, X., \& Leung, F. Y. (2018). Distress intolerance mediates the relationship between child maltreatment and nonsuicidal self-injury among chinese adolescents: a three-wave longitudinal study. Journal of Youth and Adolescence, 47(10), 22202230. https://doi.org/10.1007/s10964-018-0877-7

Khazaie, H., Zakiei, A., McCall, W. V., Noori, K., Rostampour, M., Sadeghi Bahmani, D., \& Brand, S. (2020). Relationship between sleep problems and self-injury: A systematic review. Behavioral Sleep Medicine, 19(5), 689-704. https://doi.org/10.1080/1540 2002.2020 .1822360

Kirchner, T., Ferrer, L., Forns, M., \& Zanini, D. (2011). Conducta autolesiva e ideación suicida en estudiantes de Enseñanza Secundaria Obligatoria. Diferencias de género y relación con estrategias de afrontamiento. Actas Españolas de Psiquiatría, 39(4), 226-35. https://actaspsiquiatria.es/repositorio/13/72/ESP/13-72ESP-226-235-920269.pdf

Klonsky, E. D. (2009). The funtions of self-injury in young adults who cut themselves: Clarifying the evidence for affect-regulation. Psychiatry Research, 166(2-3), 260268. https://doi.org/10.1016/j.psychres.2008.02.008

Klonsky, E. D., Muehlenkamp. J. J., Lewis. S. P., \& Walsh. B. (2011). Nonsuicidal Self-Injury. Hogrefe.

Klonsky, E. D., Oltmanns, T. F., \& Turkheimer, E. (2003). Deliberate self-harm in a non-clinical population:
Prevalence and psychological correlates. American Journal of Psychiatry, 160(8), 1501-1508. https://doi. org/10.1176/appi.ajp.160.8.1501

Laye-Gindhu, A., \& Schonert-Reichl, K. A. (2005). Nonsuicidal self-harm among community adolescents: understanding the "whats" and "whys" of self-harm. Journal Youth Adolescence, 34(5), 447-457. https://doi. org/10.1007/s10964-005-7262-z

Lewis, S., \& Arbuthnott, A. (2012). Nonsuicidal self-injury: characteristics, functions, and strategies. Journal of College Student Psychotherapy, 26(3), 185-200 https:// doi.org/10.1080/87568225.2012.685853

Liu, R. T. (2017). Characterizing the course of non-suicidal self-injury: A cognitive neuroscience perspective. Neuroscience and Biobehavioral Reviews, 80, 159-165. https://doi.org/10.1016/j.neubiorev.2017.05.026

Liverant, G. I., Kamholz, B. W., Sloan, D. M., \& Brown, T. A. (2011). Rumination in clinical depression: A type of emotional suppression? Cognitive Therapy and Research, 35(3), 253-265. https://doi.org/10.1007/ s10608-010-9304-4

Marín, M. (2013). Desarrollo y evaluación de una terapia cognitivo conductual para adolescentes que se autolesionan (Tesis doctoral). Universidad Nacional Autónoma de México, Ciudad de México.

Marín, M., Robles, R., González, C., \& Andrade, P. (2012). Propiedades psicométricas de la escala "Dificultades en la Regulación Emocional" en español (DERS-E) para adolescentes mexicanos. Salud Mental, 35(6), 521-526. http://www.revistasaludmental.mx/index.php/ salud_mental/article/view/1510/1508

Marshall, S., Tilton-Weaver, L., \& Stattin, H. (2013). Nonsuicidal self-injury and depressive symptoms during middle adolescence: a longitudinal analysis. Journal of Youth \& Adolescence, 42(8), 1234-1242. https://doi. org/10.1007/s10964-013-9919-3 
Moos, R. (1993). Coping Responses Inventory. Adult Form Professional Manual. Editorial Psychological Assessment Resources (PAR Inc.)

Muehlenkamp, J. J., \& Gutiérrez, P. M. (2004). An investigation of differences between self-injurious behavior and suicide attempts in a sample of adolescents. Suicide and Life Threatening Behavior, 34(1), 12-23. https://doi. org/10.1521/suli.34.1.12.27769

Oyanadel, C., Carrasco-Correa, H., Latorre-Nanjarí, J., Peñate-Castro, W., \& Sepúlveda-Queipul, C. (2021). Reduciendo la ideación suicida en adolescentes con terapia de perspectiva temporal. Un estudio experimental. Acta Colombiana de Psicología, 24(1), 63-71. https:// doi.org/10.14718/ACP.2021.24.1.6

Peh, C., Shahwan, S., Fauziana, R., Mahesh, M., Sambasivam, R., Zhang, Y., \& Subramaniam, M. (2017). Emotion dysregulation as a mechanism linking child maltreatment exposure and self-harm behaviors in adolescents. Child Abuse \& Neglect, 67, 383-390. https://doi.org/10.1016/j. chiabu.2017.03.013

Plener, P., Schumacher, T., Munz, L., \& Groschwitz, R. (2015). The longitudinal course of non-suicidal self-injury and deliberate self-harm: a systematic review of the literature. Borderline Personality Disorder and Emotion Dysregulation, 2(2). https://doi.org/10.1186/ s40479-014-0024-3

Robles, R., \& Garibay, S., \& Páez, F. (2006). Evaluación de trastornos disociativos en población psiquiátrica mexicana: prevalencia, comorbilidad y características psicométricas de la Escala de Expariencias Disociativas. Salud Mental, 29(2), 38-43. http://www.revistasaludmental. mx/index.php/salud_mental/article/view/1099/1097

Ross, S., \& Heath, N. (2002). A Study of the frequency of self-mutilation in a community sample of adolescents. Journal of Youth and Adolescence, 31(1), 67-77. https:// doi.org/10.1023/A:1014089117419
Santos, D. (2008). Reporte del proyecto: quiénes se autolesionan en México: Estudio Exploratorio. Documento interno. Alía.

Santos, D. (2012). Autolesión, que es y cómo ayudar (1. ${ }^{\text {a }}$ ed.). Edición de Autor.

Sociedad Mexicana de Psicología. (2007). Código ético del psicólogo (4. ${ }^{\mathrm{a}}$ ed.). Editorial Trillas.

Vallejo, A. R., \& Granados, B. E. (2017). Autoagresión y autoconcepto en adolescentes violentadas sexualmente: Una miradadescriptiva.Psicoperspectivas, 16(1),80-90.http:// dx.doi.org/10.5027/Psicoperspectivas-vol16-issue 1 -fulltext-903

Vallejo, A., Osorno, J., Mazadiego, T., \& Segura, B. (2007). Evaluación psicométrica del Inventario de Respuestas de Afrontamiento de Moos para adolescentes (CRI-Y Form), en una muestra mexicana. Revista de Educación y Desarrollo, 7, 35-40. http://www.cucs.udg.mx/revistas/ edu_desarrollo/anteriores/7/007_Vallejo.pdf

Vera, J., Bautista, G., \& Machado, F. (2018). Cultura y socialización escolar: Autolesión y género. En R. DíazLoving, I. Reyes-Lagunes y F. López-Rosales (eds.). $L a$ Psicología Social en México Vol. XVII (pp. 543-557). AMEPSO.

Walsh, B. (2006). Treating self-injury: a practical guide. The Guilford press.

Whitlock, J. L. Eckenrode, J., \& Silverman, D. (2006). Self injury behavior in a college population. Pediatrics, 117(6), 1939-1948. https://doi.org/10.1542/peds.2005-2543

Wu, R., Huang, J., Ying, J., Gao, Q., Guo, J., \& You, J. (2021). Behavioral inhibition/approach systems and adolescent nonsuicidal self-injury: The chain mediating effects of difficulty in emotion regulation and depression. Personality and Individual Differences, 175, 110718. https://doi.org/10.1016/j.paid.2021.110718 
150

You, J., Lin, M., Xu, S., \& Hu, W. (2016). Big Five personality traits in the occurrence and repetition of nonsuicidal self-injury among adolescents: The mediating effects of depressive symptoms. Personality and Individual Differences, 101, 227-231. https://doi.org/10.1016/j. paid.2016.05.057 